\title{
Sonolência excessiva diurna em alunos do último ano do curso de fisioterapia de uma faculdade privada
}

Excessive sleeping days in last year students of the physical therapy course of a private college

Sonolencia excesiva diurna en alumnos del último año del curso de fisioterapia de una facultad privada

Jéssica Barroso de Moraes $^{1 *}$, Taiane dos Santos Siebra1 ${ }^{1}$ Karla Rayane Azevedo Paz ${ }^{1}$, Carlos Antônio da luz Filho ${ }^{1}$, Karla Rakel Gonçalves Luz'1, Adriano Correia de Sousa ${ }^{1}$, Ana Beatriz Araújo Lima², Manuela Teixeira Lima1', Gabriela Teixeira Lima1', Rodrigo de Lima Souza1.

\section{RESUMO}

Objetivo: Verificar através de escalas quantos alunos do último ano do curso de fisioterapia de uma faculdade privada apresentam a Sonolência Excessiva Diurna como também avaliar uma possível associação com a ansiedade. Além disso, comparar os dados obtidos entre alunos do último ano e períodos anteriores, e identificar suas principais queixas relacionadas ao sono. Metodologia: Trata-se de uma pesquisa de campo do tipo transversal e controlada, com abordagem quantitativa onde se utilizou as escalas de ansiedade de Beck e Epworth. Participaram do estudo 93 alunos, sendo 47 alunos do último ano e 46 de anos anteriores. Resultados: A Sonolência Excessiva Diurna acometeu 31 (66\%) alunos concluintes, resultado este mais prevalente quando comparado aos alunos de períodos anteriores que obtiveram um N de $15(32,6 \%)$ estudantes. Na escala de ansiedade de Beck, ambos os grupos não apresentaram resultados relevantes. No entanto, como queixa principal relacionada ao sono houve uma prevalência da insônia em alunos do último ano, enquanto que os alunos de períodos anteriores relataram demorar mais a dormir, devido a não possuírem horários regrados para concluir suas atividades. Conclusão: Portanto, a Sonolência Excessiva Diurna foi mais prevalente nos alunos do último ano, contudo, os alunos de períodos anteriores não estão adotando hábitos saudáveis de sono, o que pode gerar repercussões negativas ao chegarem ao final do curso.

Palavras-chave: Sono, Sonolência, Estudantes.

\begin{abstract}
Objective: Verify through scales how many students of the last year of the physiotherapy course of a private faculty present the Excessive Daytime Sleepiness, as well as to evaluate a possible association with anxiety. Besides that, compare the data obtained between students of the last year and previous periods, and identify their main complaints related to sleep. Methodology: It is a field research of the type transversal and controlled with quantitative approach where the anxiety scales were used Beck and Epworth. 93 students participated in the study, of which 47 were students of the last year and 46 of previous years. Results: The Excessive Daytime Sleepiness affected $31(66 \%)$ students of the last year, a result that is more prevalent when compared to the final students who obtained an N of $15(32,6 \%)$ students. In the Beck anxiety scale, both groups did not present relevant results. However, as a primary complaint related to sleep there was a prevalence of insomnia in students of the last year, while students from previous periods reported taking longer to sleep due to not have schedules to complete their activities. Conclusion: Therefore, Excessive Daytime Sleepiness was more prevalent in students of the last year, however, the graduating is not adopting healthy habits of sleep, which can generate negative repercussions when arriving at the end of the course.
\end{abstract}

Keywords: Sleep, Somnolence, Students.

\footnotetext{
${ }^{1}$ Centro Universitário de Ciências e Tecnologia do Maranhão (Unifacema), Caxias - MA.

*E-mail: jessimoraes2017.2@hotmail.com

2 Faculdade de Ensino Superior do Piauí - (FAESPI), Teresina - PI.
} 


\section{RESUMEN}

Objectivo: Verificar através de escalas cuántos alumnos del último año del curso de fisioterapia de una universidad privada presentan la Somnolencia Excesiva Diurna, así como evaluar una posible asociación con la ansiedad. Además, comparar los datos obtenidos entre alumnos del último año y períodos anteriores, e identificar sus principales quejas relacionadas con el sueño. Metodología: Se trata de una investigación de campo del tipo transversal y controlada, con abordaje cuantitativo donde se utilizaron las escalas de ansiedad de Beck y Epworth. Participaron del estudio 93 alumnos, siendo 47 alumnos del último año y 46 de años anteriores. Resultados: La Somnolencia Excesiva Diurna acometió 31 (66\%) alumnos concluyentes, resultado este más prevalente cuando comparado a los alumnos de períodos anteriores que obtuvieron un $\mathrm{N}$ de $15(32,6 \%)$ estudiantes. En la escala de ansiedad de Beck, ambos grupos no presentaron resultados relevantes. Sin embargo, como una queja principal relacionada al sueño hubo una prevalencia del insomnio en alumnos del último año, mientras que los alumnos de períodos anteriores relataron demorarse más a dormir, debido a que no tenían horarios regulados para concluir sus actividades. Conclusión: Por lo tanto, la Somnolencia Excesiva Diurna fue más prevalente en los alumnos del último año, sin embargo, los alumnos de períodos anteriores no están adoptando hábitos saludables de sueño, lo que puede generar repercusiones negativas al llegar al final del curso.

Palabras clave: Sueño, Sonolencia, Estudiantes.

\section{INTRODUÇÃO}

Sono é um estado fisiológico complexo, funcional, reversível e cíclico, com manifestações comportamentais e fisiológicas caracterizadas por imobilidade relativa e um maior limiar de resposta aos estímulos externos (GEIB et al., 2003 e FERRO, 2005). Além da ação de liberação hormonal, o sono também assume importantes funções na preservação da memória, preservação da energia física e mental do organismo facilitando o processamento de informações e capacitando o indivíduo para desenvolver suas tarefas de forma saudável e eficaz ao longo do dia (MUGEIRO, 2011; MARTINI et., 2012).

Portanto, perturbações do sono podem acarretar alterações significativas no funcionamento físico, ocupacional, cognitivo e social do indivíduo, além de comprometer consideravelmente a qualidade de vida (MULLER e GUIMARÃES, 2007). Dormir não é somente uma necessidade de descanso físico e mental, mas também um momento para ocorrência de vários processos metabólicos que, se alterados, podem afetar o equilíbrio de todo o organismo a curto, médio e a longo prazo (CRONFLI, 2002). Embora uma pessoa precise dormir algumas horas a cada 24 horas, não é a quantidade, e sim a qualidade do sono de forma que supra as necessidades individuais (NÉRCIO, 2010).

Nos últimos anos, tem crescido a preocupação relacionada ao padrão do sono de universitários. Os chamados distúrbios do sono, especialmente a Sonolência Excessiva Diurna (SED), são as queixas mais comuns nesse grupo (BARDINI et al., 2017). Esses distúrbios subdividem-se em sete categorias: insônia, os distúrbios respiratórios relacionados ao sono, distúrbios centrais de hipersonolência, distúrbios do sonovigília, parassonias, distúrbios do movimento relacionados ao sono e outros distúrbios do sono (SATEIA, 2014).

Os distúrbios centrais de hipersonolência ou transtornos primários estão relacionados com período de iniciação ou gestão do sono ou ainda com a chamada SED, que se refere a uma propensão aumentada ao sono com uma compulsão subjetiva para dormir, tirar cochilos involuntários e ataques de sono, quando o sono é inapropriado (BITTENCOURT et al.,2005). A magnitude da SED é variada podendo e apresentar como uma sonolência leve, referida por desatenção até uma sonolência grave que podem estar presentes lapsos involuntários de sono, esquecimento e comportamento automático (NEU et al 2010).

As principais causas que levam ao desenvolvimento a SED são: qualidade ruim do sono, sono não reparador relacionado a sua pouca duração, horário de acordar desregulado, doenças médicas e neurológicas que levam a um impacto direto do sono (ansiedade, depressão, etc), medicamentos psicoativos e a presença de um transtorno primário do sono associado a hiperssonia (GIORELLI et al., 2012). 
Os estudantes se tornam um grupo bastante vulnerável para o desencadeamento desta devido à grande carga de estresse, causada pelas exigências ocupacionais, pressões familiares, compromisso com um bom desempenho acadêmico, convívio com os colegas, preocupação quanto à escolha e perspectiva profissional, longas horas de estudo e sono desordenado (MARCHI et al.,2013). Com o sono desordenado e privado o estudante tem uma tendência à sonolência excessiva durante o dia, tendo como consequência, problemas de memória, diminuição do rendimento escolar, problemas comportamentais, irritabilidade e tensão. Isso faz dos universitários um grupo que necessita de atenção em relação às suas queixas de sono para que a prevenção e diagnóstico precoce de distúrbios possam acontecer (CARDOSO et al.,2009).

Portanto, o estudo teve como objetivo verificar através de escalas quantos alunos do último ano do curso de fisioterapia de uma faculdade privada apresentam a SED, como também avaliar uma possível associação com a ansiedade. Além disso, comparar os dados obtidos entre alunos do último ano e períodos anteriores, e identificar suas principais queixas relacionadas ao sono.

\section{MÉTODOS}

O presente estudo caracterizou-se como uma pesquisa de campo, do tipo transversal e controlada com abordagem quantitativa, tendo como finalidade identificar o número de alunos acometidos por SED fazendo uma comparação entre alunos de períodos anteriores e do último ano, além de avaliar a existência de alguma associação desse distúrbio com ansiedade.

Foram avaliados 47 alunos do último ano da turma de fisioterapia de 2015.2 e 2016.1 e 46 de períodos anteriores, totalizando 93 alunos, e que tiveram como critérios de inclusão alunos devidamente matriculados no curso de fisioterapia.

A pesquisa ocorreu mediante aplicação de questionário para coleta de dados referentes aos dados pessoais como: nome, idade, sexo e estado civil e ocupação. Além disso, questionou-se as principais queixas relacionadas ao sono que os alunos referiam. E por fim aplicou-se duas escalas, uma para avaliar o nível de sonolência excessiva diurna e outra para ansiedade.

Para avaliação da Sonolência Diurna Excessiva foi aplicada a escala de Sonolência de Epworth (ESE). Essa escala contém algumas situações cotidianas, tais como chance de cochilar sentado, lendo ou assistindo à televisão, que são indicadoras da presença dessa sonolência durante o dia. A pontuação foi indicada pelo acadêmico de acordo com as seguintes instruções: 0 correspondeu a "não cochilaria nunca"; 1 correspondeu a "pequena chance de cochilar"; 2 correspondeu a "moderada chance de cochilar"; e 3 a "grande chance de cochilar". O resultado dessa escala foi somado e analisado. Pontuação entre 0 e 10 apontou ausência de sonolência, entre 10 e 16, sonolência leve; entre 16 e 20, sonolência moderada; e entre 20 e 24, sonolência severa.

Para avaliação da ansiedade foi utilizada a escala de Beck, formada por 21 itens, com quatro possíveis respostas, onde absolutamente zero obteve-se a pontuação zero, levemente teve a pontuação 1 , moderadamente teve a pontuação 2 e gravemente teve pontuação 3 , que analisaram as possíveis manifestações de ansiedade que o estudante apresentou na semana anterior. Esses 21 itens incluíram sintomas como: dormência ou formigamento, sensação de calor, tremores nas pernas, incapaz de relaxar, sensação de sufocação, nervoso, etc. Resultados de 0 a 7: grau mínimo de ansiedade, 8 a 15: ansiedade leve, 16 a 25 ansiedade moderada e 26 a 63 ansiedade severa.

Os dados foram organizados e tabulados utilizando o SPSS versão 18.0 e apresentados por meio de frequência simples e absoluta. Para verificar associação entre as variáveis foi utilizado o teste exato de qui quadrado considerando em todas as análises realizadas um nível de significância de $5 \%$.

Este projeto de pesquisa foi submetido à Plataforma Brasil conforme preconiza a resolução 466/2006 do CNS/MS, após aprovação, os alunos avaliados (ou responsáveis) foram esclarecidos sobre a pesquisa, receberam e assinaram o termo de consentimento livre e esclarecido (TCLE). CAAE: 51317115.0.0000.8007. 


\section{RESULTADOS}

A amostra foi composta por um número $(\mathrm{N})$ de 93 estudantes, sendo 47 do último ano e 46 de períodos anteriores. O sexo apresentou um número total de $65(69,9 \%)$ alunos do sexo feminino e $28(30,1 \%)$ do sexo masculino, a faixa etária mostrou que $63(67,7 \%)$ estudantes tinham idade menor que 25 anos e $30(32,3 \%)$ tinham idade superior a 25 anos, eram solteiros $75(80,6 \%)$ alunos e casados corresponderam a 18 alunos $(19,4 \%)$ do total e com relação a ocupação $22(23,7 \%)$ tinham ocupação com trabalho e 71 (76,3\%) não tinham ocupação, apenas estudavam (Tabela 1).

Tabela 1. Resultados do questionário sociodemográfico.

\begin{tabular}{|c|c|c|c|c|c|c|c|}
\hline & \multirow{2}{*}{\multicolumn{2}{|c|}{ Total }} & \multicolumn{4}{|c|}{ Grupo } & \multirow{3}{*}{$P$} \\
\hline & & & \multicolumn{2}{|c|}{ Ingressantes } & \multicolumn{2}{|c|}{ Concluintes } & \\
\hline & $\mathbf{n}$ & $\%$ & $\mathrm{n}$ & $\%$ & $\mathbf{n}$ & $\%$ & \\
\hline \multicolumn{8}{|l|}{ Sexo } \\
\hline Feminino & 65 & 69,9 & 36 & 78,0 & 29 & 61,7 & 0,082 \\
\hline Masculino & 28 & 30,1 & 10 & 21,7 & 18 & 38,3 & \\
\hline \multicolumn{8}{|l|}{ Faixa etária } \\
\hline$\leq 25$ anos & 63 & 67,7 & 29 & 63,0 & 34 & 72,3 & 0,338 \\
\hline$>25$ anos & 30 & 32,3 & 17 & 37,0 & 13 & 27,7 & \\
\hline \multicolumn{8}{|l|}{ Estado civil } \\
\hline Sem companheiro & 75 & 80,6 & 35 & 76,1 & 40 & 85,1 & 0,271 \\
\hline Com companheiro & 18 & 19,4 & 11 & 23,9 & 07 & 14,9 & \\
\hline \multicolumn{8}{|l|}{ Ocupação } \\
\hline Com ocupação & 22 & 23,7 & 13 & 28,3 & 09 & 19,1 & 0,301 \\
\hline Sem ocupação & 71 & 76,3 & 33 & 71,7 & 38 & 80,9 & \\
\hline
\end{tabular}

Fonte: Próprio autor, 2017. $\mathrm{p}^{*}=$ teste qui-quadrado

Ou seja, ambos os grupos tiveram um número maior de pessoas do sexo feminino, com faixa etária menor que 25 anos, ausência de companheiro e não tinham ocupação, apenas estudavam.

No gráfico 1 a seguir, foram organizados os resultados relativos a escala de ansiedade de Beck e a escala de Epworth para avaliar a presença da ansiedade e SED, respectivamente, nos estudantes. Os dados referentes a ansiedade mostraram que $21(44,7 \%)$ alunos do último ano são acometidos pela ansiedade, e $26(55,3 \%)$ não. Os estudantes de períodos anteriores tiveram um acometimento cujo $\mathrm{N}$ foi de $14(30,4 \%)$ alunos e ausência em 32 (69,6\%). E com relação a SED, 31 (66\%) estudantes concluintes apresentaram algum grau desse distúrbio e 16 (34\%) não apresentaram, $15(32,6 \%)$ alunos de períodos anteriores possuem a SED e $31(67,4 \%)$ não possuem.

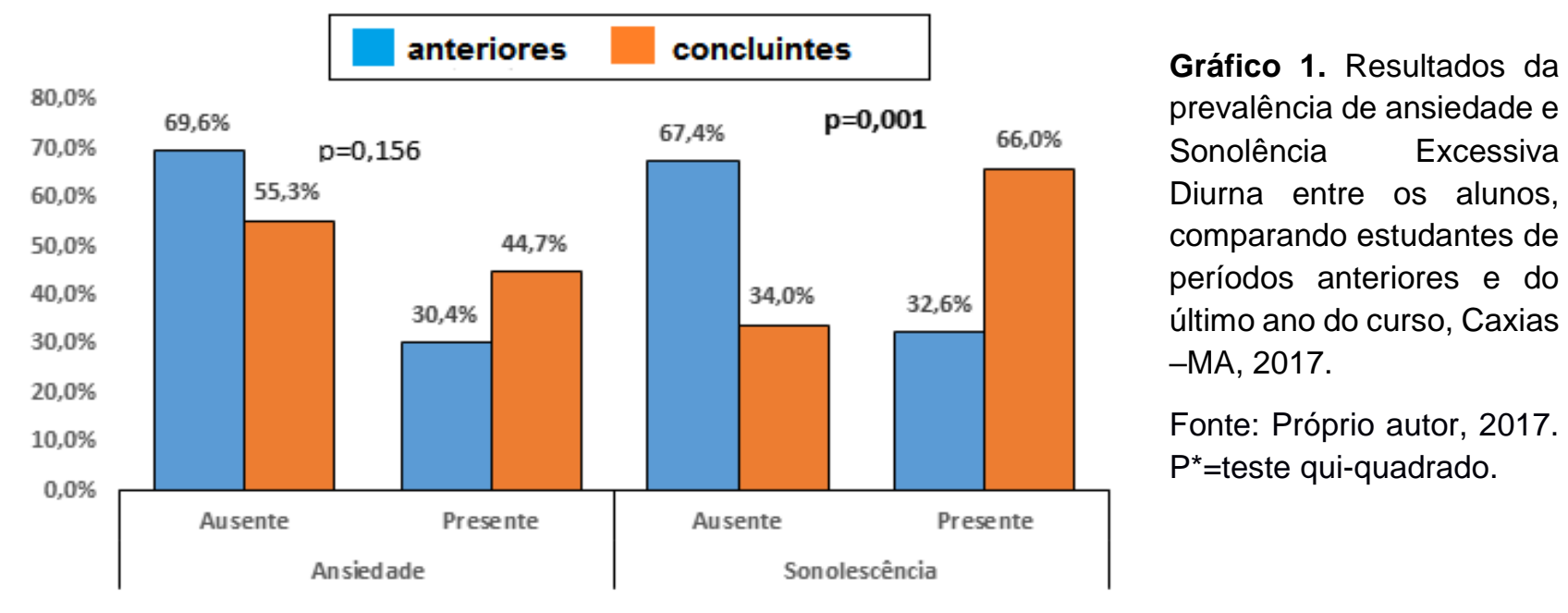


O resultado do questionário sobre queixas relacionadas ao sono (Tabela 2), mostrou que: a insônia foi referida por $6(13 \%)$ alunos de períodos anteriores e $15(31,8 \%)$ por alunos concluintes; falta de ar acometia $2(4,3 \%)$ alunos de períodos anteriores e $1(2,1 \%)$ concluinte; sonolência excessiva $3(6,5 \%)$ anteriores e 3 $(6,4 \%)$ concluintes; sono não reparador $1(2,2 \%)$ anteriores e $1(2,1 \%)$ concluinte; despertar noturno frequente $1(2,2 \%)$ anteriores e $6(12,8 \%)$ concluintes; pesadelos $2(4,2 \%)$ anteriores e $1(2,1 \%)$ concluinte; demorar dormir $13(28,3 \%)$ anteriores e $5(10,6 \%)$ concluintes.

Tabela 2. Resultado do questionário sobre queixas relacionadas ao sono.

\begin{tabular}{|c|c|c|c|c|c|c|c|}
\hline & \multirow{2}{*}{\multicolumn{2}{|c|}{ Total }} & \multicolumn{4}{|c|}{ Grupo } & \multirow{3}{*}{$\boldsymbol{P}$} \\
\hline & & & \multicolumn{2}{|c|}{ Ingressantes } & \multicolumn{2}{|c|}{ Concluintes } & \\
\hline & $\mathbf{n}$ & $\%$ & $\mathbf{n}$ & $\%$ & $\mathbf{n}$ & $\%$ & \\
\hline \multicolumn{8}{|l|}{ Queixas relacionadas ao sono } \\
\hline Insônia & 21 & 22,6 & 06 & 13,0 & 15 & 31,8 & 0,030 \\
\hline Falta de ar & 03 & 3,2 & 02 & 4,3 & 01 & 2,1 & 0,545 \\
\hline Sonolência diurna & 06 & 6,5 & 03 & 6,5 & 03 & 6,4 & 0,978 \\
\hline Sono não reparador & 02 & 2,2 & 01 & 2,2 & 01 & 2,1 & 1,000 \\
\hline Despertar noturno frequente & 07 & 7,5 & 01 & 2,2 & 06 & 12,8 & 0,053 \\
\hline Pesadelos & 03 & 3,2 & 02 & 4,3 & 01 & 2,1 & 0,545 \\
\hline Demora a dormir & 18 & 19,4 & 13 & 28,3 & 05 & 10,6 & 0,032 \\
\hline
\end{tabular}

FONTE: próprio autor, 2017. $\mathrm{p}^{*}=$ teste qui-quadrado

\section{DISCUSSÃO}

Ao longo da graduação o acadêmico a todo momento é confrontado com situações geradoras de pressão psicológica e ansiedade, esta última é definida como uma experiência emocional em função da previsão de situações futuras ou em presença de situações consideradas como desagradáveis para 0 indivíduo. (ABRANTES, 2012).

No entanto, segundo o gráfico 1 , a ansiedade não acometeu um número considerável de alunos, não apresentando também diferenças significativas entre os dois grupos. Porém, no quesito sonolência excessiva diurna, houve uma prevalência nos alunos do último ano, onde os mesmos apresentaram um acometimento de $66 \%$ com significância igual a p. 0,001 quando comparados aos alunos dos anos anteriores com porcentagem de $32,6 \%$.

Resultado similar ocorreu em pesquisa realizada numa faculdade estadual do curso de ciências biológicas, onde nesse estudo se avaliou a qualidade do sono e o nível de sonolência em alunos do ano do curso, que obteve como resultado $73,8 \%$ dos alunos com sono ruim, enquanto $63,16 \%$ com presença de SED, ambos avaliados pelas respectivas escalas de Pittsburg e Epworth. E esta equivalência é pertinente, pois uma qualidade de sono debilitada pode ter como consequência a SED. E essa relação mostrou que $66,7 \%$ das pessoas que apresentaram qualidade de sono ruim também apresentaram SED (DEPIERI et al., 2016).

Outro estudo realizado em acadêmicos da área da saúde, de uma faculdade do sul catarinense, cujo objetivo foi avaliar a prevalência da SED e seus fatores associados utilizando a escala de Epworth, percebeuse que $60,1 \%$ dos estudantes tinham dificuldades para ficar acordados em determinadas situações. Essa dificuldade apresentada poderia ter relação com o hábito de assistir televisão, fazer o uso de internet, ler e estudar na cama frequentemente. Esses itens em questão podem causar alterações no padrão normal de sono (BARDINI et al., 2017). 
Baseado nos dados contidos na Tabela 2, pode-se observar também que os alunos do último ano apresentaram uma maior incidência de insônia, com nível de significância de $\mathrm{P}=0,030$. No entanto, os alunos de períodos anteriores relataram que demoram mais a dormir, não pela dificuldade em dormir, mas por terem que cumprir obrigações das quais não tiveram tempo de fazê-las ao longo do dia, geralmente por não terem horários regrados, cujo nível de significância foi de $\mathrm{P}=0,032$.

A insônia é conceituada como a dificuldade de começar ou de manter o sono, podendo se manifestar através de despertares frequentes, de despertar prolongado durante a noite, ou de um despertar precoce, sem conformação do sono (insônia terminal). A chamada insônia psicofisiológica, ou condicionada, a forma mais frequente deste distúrbio, afeta principalmente o início do sono, enquanto a insônia terminal é mais presente em situações de depressão, mesmo que passageira (SOCIEDADE BRASILEIRA DO SONO, 2003).

As insônias podem ser primárias ou secundárias. As secundárias podem acontecer quando a insônia é consequência de uma enfermidade médica ou psíquica, outra alteração do sono ou uso excessivo de substâncias. Insônias primárias podem ocorrer devido a fatores extrínsecos ou intrínsecos, mas não são considerados como secundária a outro transtorno. Existem seis tipos de insônia primária: insônia idiopática, insônia comportamental da criança, insônia psicofisiológica, transtorno de ajustamento do sono, insônia paradoxal e higiene inadequada do sono (THROPY, 2012).

Ainda com base na tabela 2, a sonolência excessiva não foi referida como queixa principal pelos alunos, mesmo a escala de Epworth apesentando um resultado significativo para a presença da mesma na maioria dos alunos concluintes.

Sobre um estudo feito em estudantes universitários da área da saúde pode-se constatar que ao comparar os resultados de uma autoavaliação do aluno com relação a presença de SED ou não e a aplicação da escala de Epworth, percebeu-se que o número de alunos que se consideraram com SED não é o mesmo que daqueles que satisfizeram os critérios especificados pela escala, porém os indivíduos que acreditaram ter SED apresentam significativamente maior prevalência de SED (segundo tal escala) (BARDINI et al., 2017).

A SED apresenta várias causas possíveis, porém a mais comum é a síndrome do sono insuficiente, na qual os pacientes têm um sono cronicamente privado pelos compromissos de trabalho e outras obrigações sociais (CHOKROVERTY, 2009).

O sono insuficiente tem um grande impacto no bem-estar da população, afetando a capacidade de funcionar em condições ótimas, aumentando perturbações psiquiátricas, exacerbando condições crônicas, rompendo tratamentos médicos, além de contribuir para uma inabilidade social associada a uma doença crônica (STRINE e CHAPMAN, 2005).

Os questionários utilizados neste estudo foram aplicados por diversas pesquisas demonstrando a boa aplicabilidade de tais ferramentas para avaliar subjetivamente as informações de caráter qualitativo e quantitativo do sono e sonolência diurna excessiva. Esses instrumentos são acessíveis, simples, de fácil entendimento e preenchimento rápido, ao contrário da polissonografia, que é o padrão ouro para avaliação da qualidade do sono e seus distúrbios, necessitando de um espaço físico adequado, recursos humanos treinados, o que exige um investimento financeiro elevado, restringindo sua acessibilidade a alguns centros clínicos do país (BETOLAZI, 2008).

\section{CONCLUSÃO}

Conclui-se que a SED acometeu mais os alunos concluintes, o que confirma a hipótese de que este público está mais sujeito a apresentar esse distúrbio. No entanto, a ansiedade não foi o fator causador do mesmo nos alunos, demonstrando assim, que o desenvolvimento da SED pode estar relacionada a diversas outras causas como a insônia. A insônia foi a queixa principal mais referida pelos alunos do último ano enquanto que os de períodos anteriores relataram demorar mais a dormir, devido a não possuírem horários regrados para concluir suas atividades ainda durante o dia. Portanto, a insônia presente nos alunos concluintes pode ser resultado de uma cronicidade desse horário para dormir quando ainda estavam no início do curso, gerando 
essa dificuldade para dormir quando chegaram ao último ano da graduação. Logo, os estudantes precisam organizar seus horários para ter uma noite de sono adequada.

\section{REFERÊNCIAS}

1. ABRANTES NNF. Trabalho e estudo: uma conciliação desafiante. Campina Grande, REALIZE Editora, 2012

2. BARDINI $R$ et al. Prevalência de sonolência excessiva diurna e fatores associados em adolescentes universitários do sul catarinenese. Arq. Catarin Med. 2017; jan-mar; 46(1): 107-124.

3. BERTOLAZI NA. Tradução, adaptação cultural e validação de dois instrumentos de avaliação do sono: escala de sonolência de Epworth e Índice de qualidade do sono de Pittsburgh. [Dissertação para obtenção do título de mestre] apesentada a Universidade federal do Rio Grande do Sul,Faculdade de medicina, Porto Alegre, 2008.

4. BITTENCOURT LR et al. Sonolência excessiva. Rev Bras Psiquiatr. 2005;27(Supl I):16-21.

5. CARDOSO HC et al. Avaliação da qualidade do sono em estudantes de Medicina. Rev Brasileira de educação médica. $2009 ; 33$ (3) : 349-355.

6. CHOKROVERTYS. Sleep disorders medicine: basic science, technical considerations, and clinical aspects Overview of sleep \& sleep disorders. Indian J Med Res.2010; Feb;131:126-40.

7. CRONFLI RT. A importância do sono. Cérebro\&mente, Campinas, dez/2002.

8. DEPIERI NB et al. Qualidade do sono e sonolência entre universitários formandos. Arquivos do MUDI. 2016; 20(2),33-42.

9. FERRO CV. Correlação dos distúrbios do sono e parâmetros subjetivos em indivíduos com fibromialgia. [monografia de graduação]. Cascavel: Unioeste; 2005.

10. GEIB LT. Sono e envelhecimento. R. Psiquiatr. RS. 2003; 25'(3): 453-465.

11. GIORELLI A et al. Sonolência excessiva diurna: aspectos clínicos, diagnósticos e terapêuticos. Rev Bras Neurol.2012; 48 (3): $17-24$.

12. MARCHI K et. al. Ansiedade e consumo de ansiolíticos entre estudantes de enfermagem de uma universidade pública. Rev. Eletr. Enf. $2013 \mathrm{jul} / \mathrm{set} ; 15(3): 731-9$.

13. MARTINI M et al. Fatores associados à qualidade do sono em estudantes de Fisioterapia. Fisioter Pesq. 2012;19(3):261-267.

14. MUGEIRO MJ. Qualidade do sono nos idosos. [Dissertação de mestrado] Instituto Politécnico de Viseu, Escola Superior de Saúde de Viseu, 2011.

15. MULLER MR, GUIMARÃES SS. Impacto dos transtornos do sono sobre o funcionamento diário e a qualidade de vida. Estudos de Psicologia. Campinas. 2007; 24(4), 519-528.

16. NÉRCIO TR. Qualidade do sono e hábitos de estudo em jovens institucionalizados. [dissertação de mestrado]. Porto/Portugal: Universidade Fernando Pessoa; 2010.

17. NEU D et al. Clinical complaints of daytime sleepiness and fatigue: how to distinguish and treat them, especially when tehy become excessive or chronic? Acta Neurologica. 2010; 110(1):15-25.

18. SATEIA MD. International Classification of Sleep Disorders, $3^{\circ}$ Edition, Contemporary Reviews in Sleep Medicine, 2014.

19. SOCIEDADE BRASILEIRA DO SONO. I Consenso Brasileiro de Insônia. Hypnos, J Clin Exp Sleep Res; 2003.

20. STRINE T, CHAPMAN D. Associations of frequent sleep insufficiency with healthrelated quality of life and health behaviors. Sleep Medicine, Atlanta, USA. 2005; 6, 23-27.

21. THORPY, MJ. Classification of Sleep Disorders. Neurotherapeutics. 2012 Oct; 9(4): 687-701. 